\title{
Tweening Grammars: Deformation Rules for Representing Change between Discrete Geographic Entities
}

\author{
Tae H. Kim ${ }^{1}$ and Thomas J. Cova ${ }^{2}$ \\ ${ }^{1,2}$ Department of Geography, University of Utah, \\ Salt Lake City, UT 84112 \\ Tel: (801) 581-8218 \\ Fax: (801) 581-8219 \\ Email tae.kim@geog.utah.edu
}

\begin{abstract}
:
Data concerning dynamic geographic processes are commonly captured and stored as discrete temporal snapshots. Snapshot data often requires interpolation to compensate for large data collection intervals.

Metamorphosis (morphing or tweening) is the study of how an object changes over time. Tweening was developed with basic transformations in mind rather than any particular underlying geographic process that might be driving the change (e.g. wildfire). This paper describes a means for making the interpolation between two snapshots more effective and efficient by including process-informed rules to guide the tweening process. Background is presented on geographic transformations, map animation, and tweening classifications. A rule-based tweening procedure is presented that is based on a set of deformation rules including a case study for wildfire snapshots. A hybrid approach using a rule-based and medial axis transformation is introduced. The paper concludes with a discussion of the strengths and limitations of the approaches.
\end{abstract}

\section{Introduction}

Data about dynamic geographic processes (e.g. wildfire spread) are generally captured and stored as discrete temporal "snapshots". This is largely due to the limited availability of sensing technology to record and store continuous data. When data is collected, the information pertaining to the what, where and when of an event are important in conducting analyses and reconstructing events (Yuan 1999; Peuquet 2002). Shapes in the form of vector-based graphics are commonly produced from raster-based graphics regarding the geographic phenomenon of interest. The data generally contains the boundary and extent of the phenomenon at a given point in time. When performing analysis and producing visual representations of change, interpolation between snapshots is required. This is especially the case when there are infrequent or inconsistent data collection intervals (Kim et al., in press).

Metamorphosis is the study of change of an object over time or between two objects. Tweening is another term for a metamorphic process that can be traced back to the origins of computer animation when algorithms were developed to reduce the amount of frames an animator had to produce (Catmull 1978; Reeves 1981; AvRuskin et al. 2004). Tweening is short for in-betweening where intermediate frames are generated for both raster and vector data to produce a visually continuous animation. Tweening can be applied to tint (color), motion (translation) and shape (transformation) of an object (AvRuskin et al. 2004). Generally, a time element is explicitly indicated in the process, 
represented as intermediate frames or discrete number of steps between the source and target shapes.

Morphing is another term for tweening and generally describes the same process. Although some researchers differentiate tweening and morphing (Winnemöller and Bangay 2001) by stating that tweening is the metamorphosis of a single object and morphing relates to the metamorphosis of one object in to another object, these definitions do not demarcate a clear distinction in the literature. When two shapes are merged to produce a new shape, this is commonly referred to as shape blending. Shape blending can be best visualized mentally by imagining two objects adjacent to one another but not touching. Then visualizing a bridge forming to connect the two shapes, this is a standard example of shape blending. As the term indicates, shape blending blends two or more shapes into a new single shape.

Ordinary (typical or standard) tweening has limitations in producing meaningful mappings between two specified snapshots (Glassner 2003). That is to say a limitation arises in realistically representing a geographic process that is being represented (Johnson 1999) such as wildfire spread (Kim et al. in press). A better animation can be produced by manually assigning shape hints (or guides) to map correspondence between points on the source and target shapes. This can be a labor intensive process, especially when dealing with time-series data containing numerous snapshots. A more thorough approach to interpolating wildfire spread is to apply a process model (Rothermel 1972; Finney 1998) which takes into consideration realistic variables (e.g. slope, aspect, fuel, wind and moisture). This requires many data layers and detailed knowledge about the process being modeled. An alternative approach is required to provide more general tools for representing change between geographic entities in a geocomputational framework employing a less labor intensive approach and detailed knowledge of a geographic process.

In order to reduce the user input requirements and provide a more robust set of tools for producing more realistic tweens, rules defining the tweening process can be developed. Rules are generally simple procedures that are assigned to the behavior of shapes and or entities and have been applied to the study of the shape transformation through shape grammars since the 1970's (Stiny and Gips 1972). For instance, one can visualize a transformation rule requiring that all isosceles triangles be rotated 90 degrees clockwise about the point at the angle containing the two equal sides. The focus in most spatial modeling is generally on the before and after conditions, leaving the intermediate frames as a mental visualization with only the initial and final states displayed. A tweening rule and animation can externally represent the process of change, thus helping to visually communicate the transformation rules and relationships of discrete entities.

The goal of this project is to make the interpolation between two snapshots of discrete entities more effective and efficient. The outcome of this research is a set of generalized rules to better represent change in a geographical context and possibly improve and inform metamorphosis techniques in other subsequent disciplines. The paper proceeds with a general background on common geographic information systems transformations, map animation, tweening and classifications of research literature. New methods and their limitations are discussed in section 3. It begins with a taxonomy of the different source and target shape relationships followed by an exploration of a semi-automatic correspondence and trajectory pseudocode as well as path and rate variations. The results 
section presents a hybrid tweening procedure using a medial axial and semi-automatic transformation approach followed by the application of to a wildfire case study. The paper concludes with a discussion of the results.

\section{Background}

This section reviews the literature in the fields of animation, computational geometry, computer graphics and geography pertaining to tweening. The purpose of the review is to identify key concepts which may be applicable to this particular research and to highlight gaps in existing research. A secondary goal is to identify areas which have been investigated with little success and are better to avoid. Subsection 2.1 reviews the research conducted in GIS and map animation. In subsection 2.2 we proceed with an overview of tweening that reviews problems that researchers focusing on metamorphosis have been grappling with for decades. Subsection 2.3 proposes a taxonomy of the tweening literature to organize and categorize the many different approaches. It is through this process that clarification of exis ting methods will be achieved.

\subsection{GIS Transformations}

Geographic Information Systems (GIS) utilize numerous transformation techniques including the standard affine transformations of translation, rotation, mirror and scale, as well as the curvilinear transformation techniques with a higher order transformation where straight lines are not necessarily maintained (Goodchild 1984). An example of a curvilinear transformation is rubber-sheeting in GIS for geo-rectifying data layers and maps where control points represent corresponding points selected by an analyst. This is similar to manipulating an underlying mesh or triangulated-irregular network using several control points. Some GIS packages can handle time-series data, while map animation has been a research topic within a computer environment since the late 1950's (Thrower 1959; Lobben 2003). Still, most GIS do not have the functionality to handle the metamorphosis of snapshot data. Johnson (1999) identified problems associated with tweening for the TimeMap project, but there has since been no progress report.

A concept of spatiotemporal helixes has been introduced by Stefanidis et al. (2003) to account for change in snapshot data but their focus is more concerned with the trajectory of shape centroids and the spatial difference of the two shapes. Their study does not indicate how one shape transforms into another. The storage of discrete time slices and how they are stored in cartographic representation have been a prior focus in GIS research (Langran 1993; Yuan 1999), but again there has been little focus on metamorphic processes between time slices that are geographic in nature.

\subsection{Metamorphosis}

This section reviews metamorphosis (tweening) approaches and their limitations. Specific problems of tweening relating to correspondence, vertex intersection and boundary constraints are discussed. In addition, classifications of the different source and target shape relationships are determined to understand the interpolation between two snapshots of discrete entities to make more effective and efficient tweens.

\subsubsection{Correspondence}

The ideal situation is to tween a source shape to a target shape consisting of the same amount of vertices and which has some form of shape persistence. The transformation can be done in either a clockwise or counter-clockwise direction without much affect in the metamorphosis (Figure 1). According to some authors (Reeves 1981; Gotsman and 
Surazhsky 2001), there is no successful solution to the automatic mapping or correspondence of vertices problem. Thus, user input is always required to produce realistic tweens to maintain a user identified shape persistence and the relationship between features.
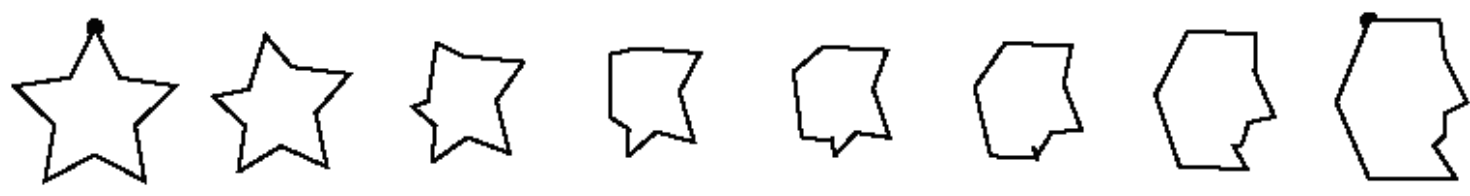

Figure 1 DMorph (Glassner 2003)

Once correspondences between source and target points are manually assigned, there exist many approaches to tweening. There are shape interpolation approaches that affect the vertices of shapes through reduction in to triangulated irregular networks, skeletons and grids (Lee et al. 1998; Gueziec 1999). Many of these techniques can be applied to 2D and 3D images and shapes and they are widely utilized for facial and character modeling and animation (Noh and Neumann 1998). The metamorphosis can be in the form of a uniform or non- uniform transformation (Lee et al. 1998) using simple linear interpolation or curve-based interpolation. In these circumstances, the vertex moves along "movealong" some interpolated path (Reeves 1981).

Two shapes may not always have the same amount of vertices. When the target shape has more vertices than the source shape, one approach is to add additional vertices after the last vertex of the source shape has been mapped to the target shape. Another approach is to count the difference in the number of vertices and evenly insert extra vertices called Steiner points (extra points) in-between existing vertices of the source shape (Figure 2).

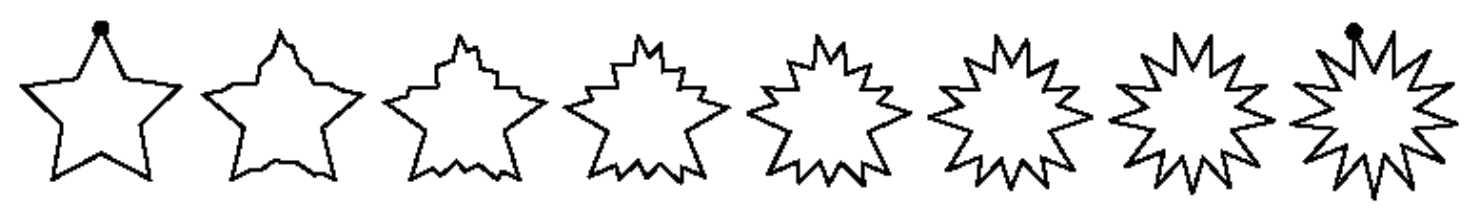

Figure 2 A morph where the system inserted new vertices into both shapes so they both had the same number of points (Glassner 2003).

Andrew Glassner developed DMorph (Glassner 2003) as an automatic technique for morphing polygonal objects without the same quantity of vertices and faces. The algorithm only works on convex objects, meaning objects without any indentations or undercuts. Complicated shapes are generally concave and this is the case for most geographic phenomena represented as shapes.

\subsubsection{Trajectory: Intersection-free metamorphosis}

Trajectory of correspond ing vertices or the movement path is another problem which requires user input to inform the metamorphosis (Gotsman and Surazhsky 2001). When tweening is applied to source and target shapes that are very different, it is common to get a mangle of vertex crossings and lines during the tween (Figure 3 ). This is due to the lack of heuristics to inform the shape tweening process (Glassner 2003) permitting middle vertices to pass through one another to arrive at there target positions. This is because 
each vertex path is determined independently of other vertex paths and uninformed by a geographic process (Shapira and Rappoport 1995).

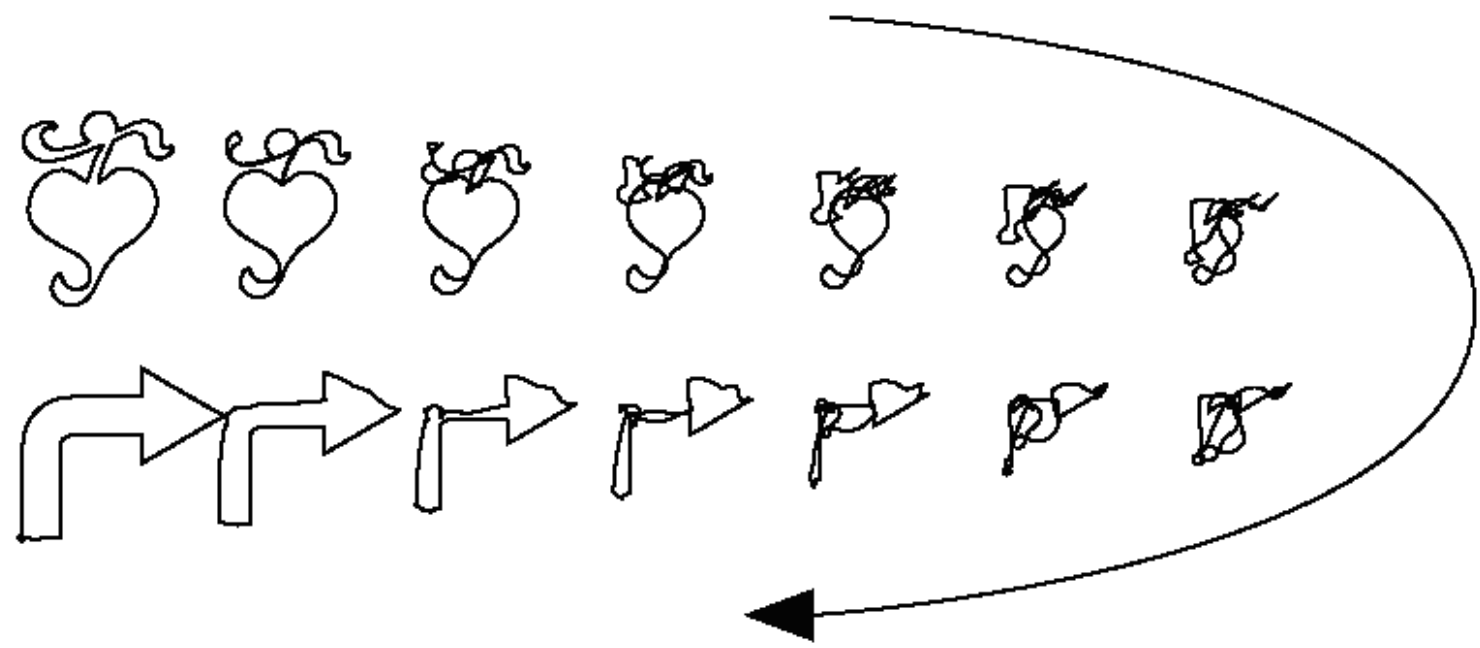

Figure 3 Automatic morphs often get tangled up in the middle as vertices pass through each other en route to their destinations. (Glassner 2003)

Figure 4 shows a guaranteed intersection-free polygon morphing using triangulated polygons as discussed by Gotsman and Surazhsky (Gotsman and Surazhsky 2001). Another solution is an edge-angle interpolation where the interpolated entities are lengths and angles instead of vertices (Sederberg et al. 1993). This approach is still problematic because entity relationships apply only to the adjacent lengths and angles.

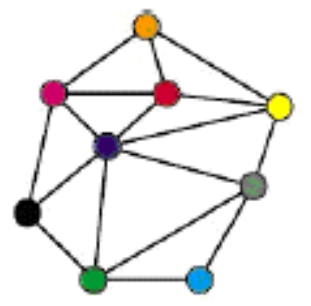

(a)

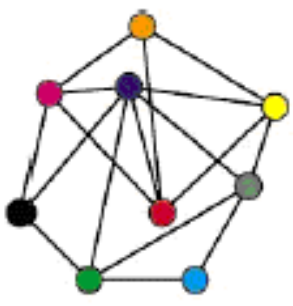

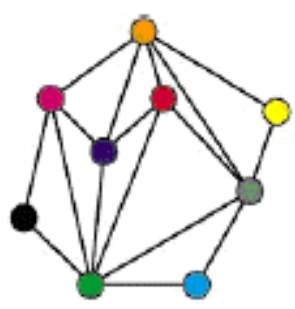

(b)

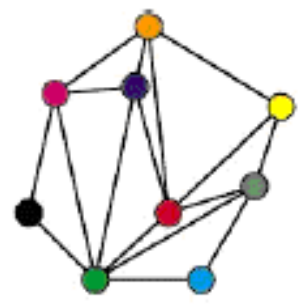

Figure 4. (a) Corresponding point sets (correspondence denoted by colored vertices) with incompatible triangulation. (b) Same point sets with compatible triangulation. (Gotsman and Surazhsky 2001)

\subsubsection{Skeletons and Medial Axis Transformation}

Decomposing a shape into a skeleton is a common procedure preceding shape morphology and it is similar to the process of producing TINs (Kirkpatrick and Radke 1985). It describes the internal structure of planar point sets. An approach to skeletal decomposition of a shape which sometimes uses a geographic metaphor is a medial axis technique (Blum 1967; Blum and Nagel 1978; Kerckhove 1999; Katz and Pizer 2003). Figure 5A represents water ripples traversing through each other, and figure 5B shows expanding perimeters that block each other and meet along a line representing the medial 
axis. Figure 5C represents contractions and expansions of a triangular outline terminating along an upside down ' $Y$ '-shaped medial axes. One can imagine that the triangle in figure $5 \mathrm{C}$ represents a grass fire boundary propagating towards the interior and exterior of the triangle. It produces a medial axis skeleton of the source shape. Obtaining the medial axis of a source and target shape provides a fruitful springboard for formulating realistic geographic metamorphosis processes (Che et al. 2004; Yang et al. 2004) and will be discussed further in the methods section.

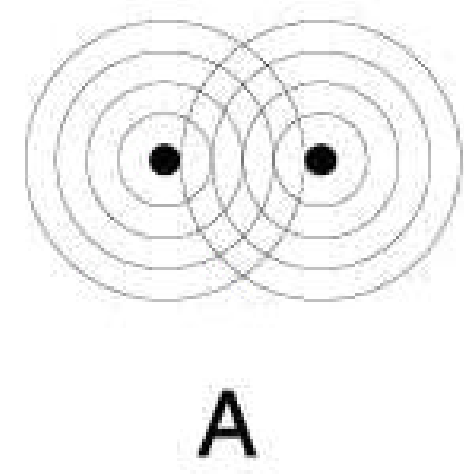

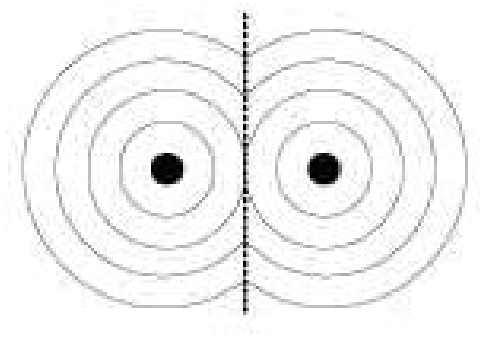

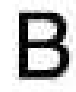

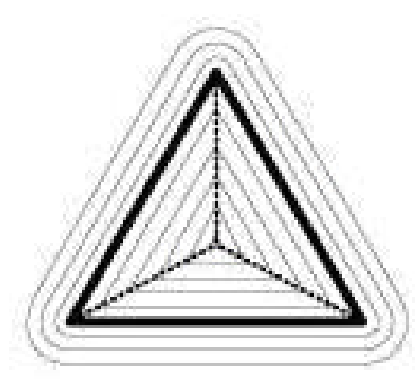

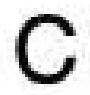

Figure 5. Harry Blum's medial axis transformation from “An Intuitive Explanation of medial Axis Transformation” (http://www.ipc.kit.ac.jp/ gert/medax/medax.html).

\subsubsection{Metamorphosis Taxonomy}

There is much literature on metamorphosis, but there is little literature directly addressing tweening rules specific to a geographic process. Table 1 categorizes research papers by its primary research application. As indicated in the table, the majority of research has been conducted to inform the field of animation and followed closely by computer-aided design (CAD) research. Animation researchers have been mostly concerned with reducing the amount of time to produce in-between frames for animations. Their approach and concerns are for more realistic tweens, but they have not focused on tweens directly related to geospatial processes.

Table 1. Taxonomy based on research application.

\begin{tabular}{|l|l|}
\hline Animation (Facial) & $\begin{array}{l}\text { (Lee et al. 1996; Lee et al. 1998; Noh and Neumann 1998; } \\
\text { Winnemöller and Bangay 2001) }\end{array}$ \\
\hline & $\begin{array}{l}\text { (Martyn; Blum 1967; Blum and Nagel 1978; Reeves 1981; } \\
\text { Kirkpatrick and Radke 1985; Shapira and Rappoport 1995; } \\
\text { Noh and Neumann 1998; Bradley and Stuart 2001; Di Fiore et } \\
\text { animation (Character, 2001; Gotsman and Surazhsky 2001; Van den Bergh et al. } \\
\text { 2D/Shapes) }\end{array}$ \\
$\begin{array}{l}\text { 2002; Glassner 2003; Torsello and Hancock 2004; Yang et al. } \\
\text { 2004) }\end{array}$ \\
$\begin{array}{l}\text { CAD, CAAD, MCAD: } \\
\text { ID, Arch, Mech Eng., } \\
\begin{array}{l}\text { Object Morphing, } \\
\text { 3D/Shapes }\end{array}\end{array}$ & $\begin{array}{l}\text { Pasko et al. 1999; Gotsman and Surazhsky 2001; Surazhsky } \\
\text { et al. 2001; Winnemöller and Bangay 2001; Pasko et al. 2002; } \\
\text { Van den Bergh et al. 2002; Glassner 2003; Che et al. 2004) }\end{array}$ \\
\hline
\end{tabular}




\begin{tabular}{|l|l|}
\hline $\begin{array}{l}\text { Deformation methods } \\
\text { (Skeleton, TIN) }\end{array}$ & (Kirkpatrick and Radke 1985) \\
\hline Images (General) & (Lee et al. 1996; Surazhsky et al. 2001) \\
\hline Medical Imaging & $\begin{array}{l}\text { (Shih et al. 1998; Pasko et al. 1999; Treece et al. 1999; } \\
\text { Surazhsky et al. 2001) }\end{array}$ \\
\hline Topography & (Surazhsky et al. 2001) \\
\hline
\end{tabular}

The second table (Table 2) shows the interdisciplinary scope of this problem and that many researchers are interested in tweening. The table categorizes the research papers into the fields of the lead authors. As one can see from the table, there is little research focus by researchers interested in geospatial phenomena (Table 2).

Table 2. Discipline of lead author of the publication.

\begin{tabular}{|l|l|}
\hline Computer Graphics, & (Martyn; Blum 1967; Blum and Nagel 1978; Catmull 1978; \\
Science, Animation & Reeves 1981; Kirkpatrick and Radke 1985; DeCarlo and \\
& Metaxas 1995; Shapira and Rappoport 1995; Lee et al. 1996; \\
& $\begin{array}{l}\text { DeCarlo and Metaxas 1998; Gao and Sederberg 1998; Lee et } \\
\text { al. 1998; Pasko et al. 1999; Johan et al. 2000; Bradley and } \\
\text { Stuart 2001; Gotsman and Surazhsky 2001; Winnemöller and } \\
\text { Bangay 2001; Pasko et al. 2002; Van den Bergh et al. 2002; } \\
\text { Glassner 2003; Torsello and Hancock 2004; Yang et al. 2004) }\end{array}$ \\
\hline Architecture \& & (Stiny and Gips 1972; Bertalmio et al. 1998; Shih et al. 1998; \\
Engineering & Treece et al. 1999; Treece et al. 2001) \\
\hline Mathematics & (Surazhsky et al. 2001; Che et al. 2004) \\
\hline
\end{tabular}

\section{Methods}

\subsection{Source and Target Shape Relationships}

Categorization of the different shape-to-shape relationships was conducted to identify several types of source and target shape relationships. This includes nested, overlapping, adjacent and exclusive relationships between shapes. In addition, a one-to-many and many-to-one ( $n$ to $x * n$ ) relationship of shapes was identified. The directionality of the change can be bi-directional for all the source and target shape relationships. 


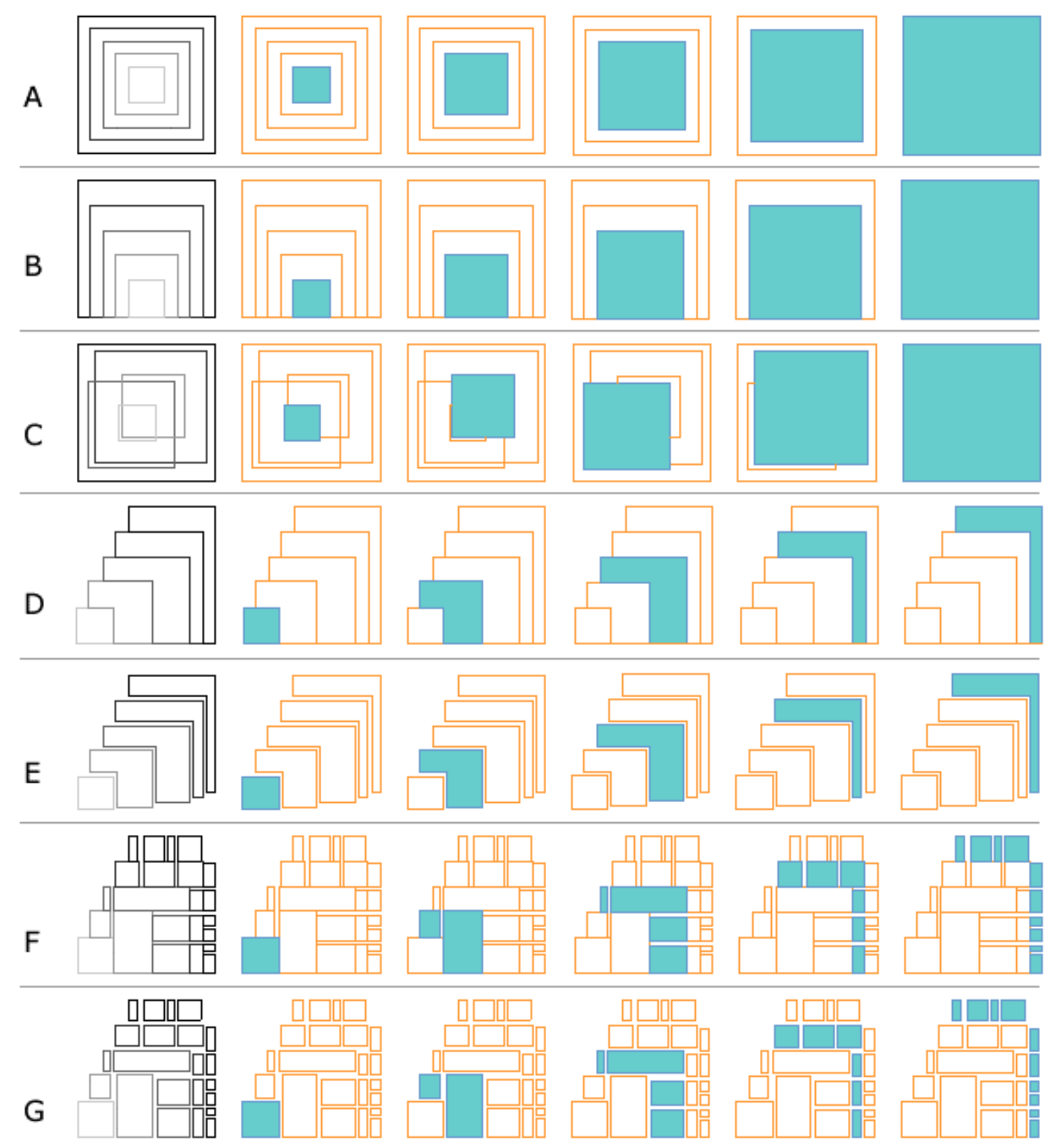

Figure 6. Source and target shape relationships

Figure 6 shows seven different spatial relationships between source and target shapes. Each row displays the progression of five intervals $\left(t_{0}, t_{1}, t_{2}, t_{3}\right.$, and $\left.t_{4}\right)$ starting from the second image from the left and the first image displaying the layered outlines of five snapshots. The top two rows show nested shapes where Figure 6A does not include shared features and Figure 6B does. Overlapping shapes with shared features are shown in Figure 6C, and there can be no overlapping shapes without shared features in a twodimensional space. This is followed by adjacent shapes (with shared features) (Figure $6 \mathrm{D}$ ) or exclusive shapes (without shared features) (Figure 6E) and $n$ to $x^{*} n$ shapes (one to many or many to one) with shared features(Figure 6F) and without (Figure 6G) shared features.

In order to reduce the complexity and scope of this research and to avoid preexisting limitations with correspondence and trajectory between shapes, the types of source and 
target shape relationships were narrowed. The source and target shape should be nested within one another or share common features. Figure 7 shows the five types of shape relationships that are further explored in this research, including: 1) nested without shared features (Figure 7A), 2) nested with shared features (Figure 7B), 3) adjacent shapes with shared features (Figure 7C), 4) overlapping (Figure 7D) and 5) a $n$ to $x^{*} n$ relationship with shared features (Figure 7E).

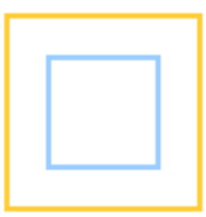

A

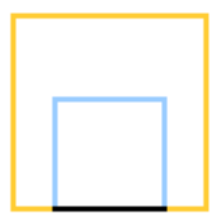

B

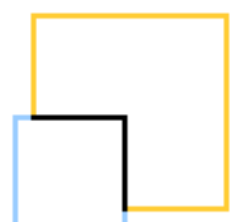

C

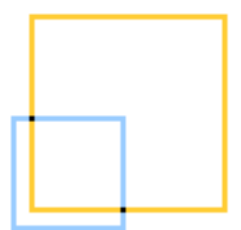

D

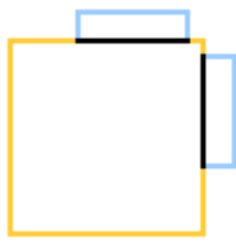

E

Figure 7. Narrowed shape relationships.

\subsection{Tweening Grammars : Correspondence and Trajectory}

Figure 8 displays a set of mid-tweened shapes based on the various trajectories of corresponding vertices. This is represented in each top and bottom lettered set in figure 8 with the mid-tween shape and the trajectory of the vertices represented below each example. For the refined set of shape relationships, tweening grammars can be designed to inform the movement of corresponding points. Such grammars avoid undesired correspondence and the intersection of vertices resulting in a polygon that is not filled with a color (Figure 8A and 8B). Empty polygon fills are generally an artifact of the default paint or draw functions in programming languages which considers only a singlesided polygon or accounts for only one filled polygon at a time. Figure $8 \mathrm{C}$ presents a case where a rotation occurs in a clockwise manner in regards to vertex correspondence. A rule can be formulated to prevent the intersection of vertices during the tweening process by using the shortest path between corresponding point sets (Figure 8D). Also, constraints addressing the bound ing perimeter of the shapes provide more realistic tweens between source and target shapes (Figure 8E).

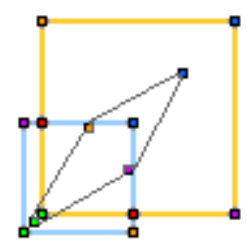

A

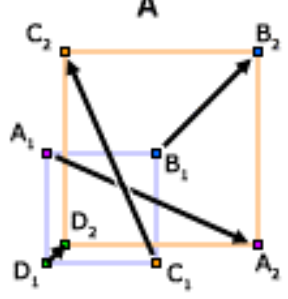

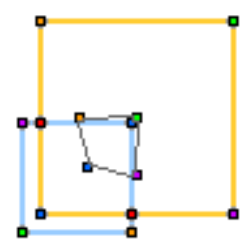

$\mathbf{B}$

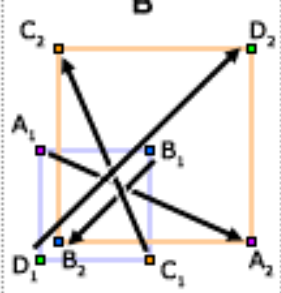

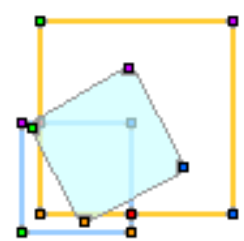

C

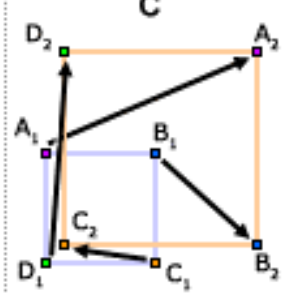

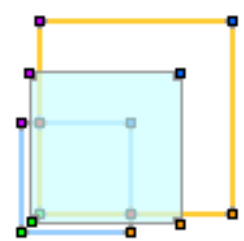

D

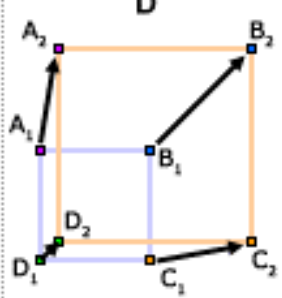

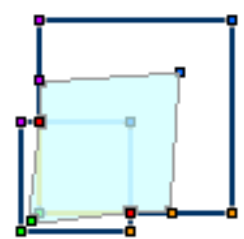

E

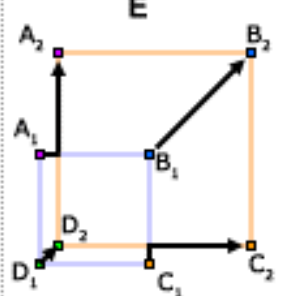

Figure 8. Correspondence and trajectory variations and the resulting mid-tween shape.

A tweening grammar in pseudocode format is presented below using a shortest path from the source and target vertex with constraints (Figure 9). The purpose of the pseudocode 
is to assign a semi-automated correspondence of vertices, prevent the mangling of vertices and insure that bound ing perimeters of the source and target shapes are not crossed.

1.Determine the joined boundary of the two shapes (Figure 9B).

2.Ensure that points travel within or along the boundary of the two shapes.

3.Determine shared features.

4.Add Steiner points on shared vertices (Figure 9C).

5.For every point on the source object.

a. Assign correspondence using a shortest-distance assumption from a point on the source object to a point on the target object (Figure 9D).

i. Ensure that the source and target vertex are not the same point.

ii. If overlapped shapes

1. Ensure that both source and target vertices within overlapped regions do not have correspondence.

6.A source point (which is mutually exclusive to a target shape) must not correspond to a Steiner point.

7.Non-corresponded points on the source object become points on a line.

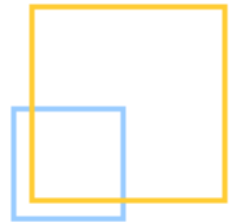

A

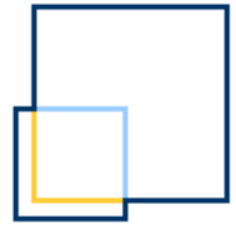

B

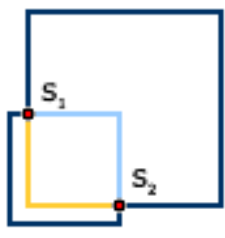

C

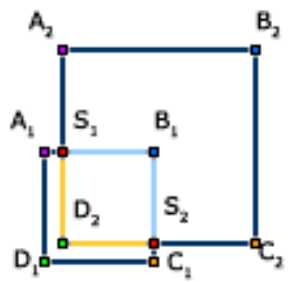

D

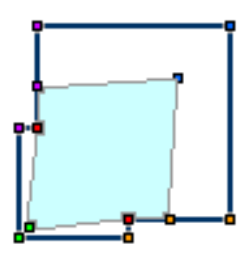

$\mathbf{E}$

Figure 9. Example of correspondence with constraints.

Figure 9E shows an intermediate shape at approximately halfway through the tween of the source and target shapes. It is important to note that the portion relating to correspondence in the above tweening grammar may not work with every source and target shape combination. As indicated in previous sections, the problem of automatic tweening which preserves features has not been successfully solved, and is not the goal of this research. The semi-automatic correspondence method is explored here to simply prevent intersection of vertices and to insure the movement of vertices is within the boundary of the shapes.

The mid-tweens for the different source and target shape relationships are shown in figure 10 represented by the shape with grey segments with a light blue fill. In addition, there are two shapes represented by light blue and yellow colored segments. They are the source and target shapes that the mid-tweens are derived from and where the colors are simply meant to distinguish the two shapes. Figure 10A shows the mid-tween for a nested shape relationship and figure 10B shows a mid-tween for nested shapes with shared features. A mid-tween for adjacent shapes with shared features is displayed in figure 10C and a mid-tween for an overlapping relationship is presented in figure 10D and a one-to-many shape relationship $\left(n\right.$ to $\left.x^{*} n\right)$ is shown in figure $10 \mathrm{E}$. 
A

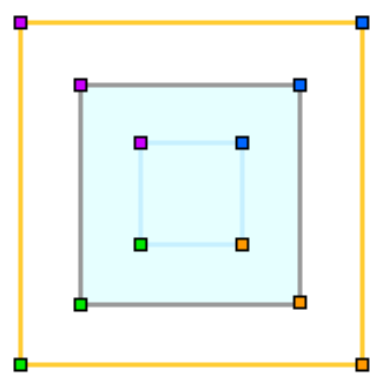

D

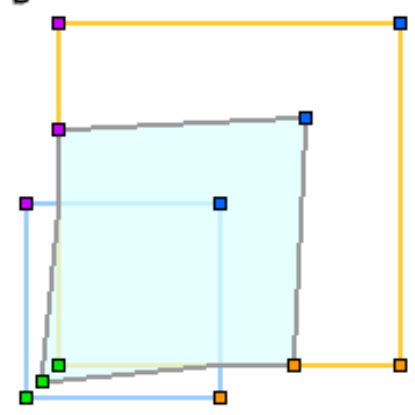

B

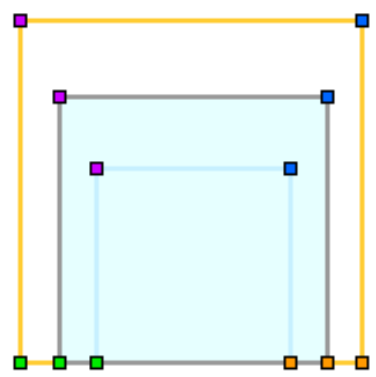

E

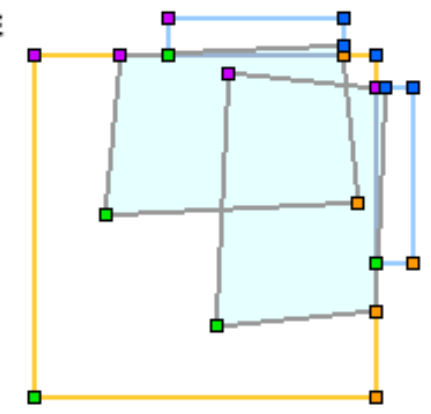

C

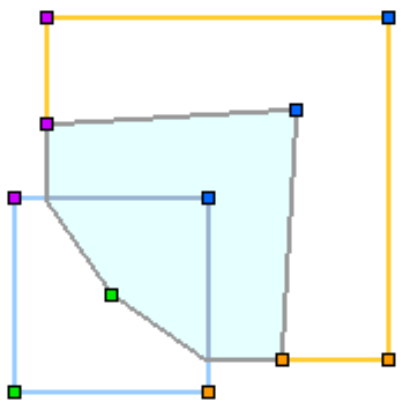

MID-TWEENS FOR DIFFERENT SHAPE RELATIONSHIPS
A Nested
B Nested with shared features
C Adjacent (with shared features)
D Overlapping
E One to Many

Figure 10. Mid-tweens for different source and target shape relationships.

\subsection{Trajectory: Path}

A vertex can move along the source to target location through various paths while maintaining intersection free movement. As indicated above, it is important that the moving vertices stay within the defined boundaries. Table 3 shows a matrix of the different rate and path combinations for vertex movement to produce various tweens that may represent different types of processes.

Table 3

\begin{tabular}{|c|c|c|c|}
\hline \multicolumn{4}{|c|}{ Time-Space Matrix for tweening of geographic processes } \\
\hline \multirow{2}{*}{\multicolumn{2}{|c|}{$\begin{array}{l}\text { Vertex movement interpolation } \\
\text { between two corresponding } \\
\text { points. }\end{array}$}} & \multicolumn{2}{|c|}{ PATH } \\
\hline & & Pre-Defined & Dynamic \\
\hline \multirow[t]{2}{*}{ RATE } & Constant & $\begin{array}{l}\text { Straight Line, } \\
\text { Curves \& Poly lines } \\
\\
\text { (Linear path \& } \\
\text { Constant rate) }\end{array}$ & $\begin{array}{c}\text { Non-linear path \& Constant } \\
\text { rate }\end{array}$ \\
\hline & Non-Constant & $\begin{array}{c}\text { Straight line, Curves } \\
\text { \& Poly lines } \\
\text { \& Variable rate }\end{array}$ & $\begin{array}{c}\text { Non-linear path \& Variable } \\
\text { rate }\end{array}$ \\
\hline
\end{tabular}


The pre-defined path that a vertex travels can be defined by a rule such as a straight line shown in figure $11 \mathrm{~A}$ and would be the simplest path for correspondence. The path can be a poly line as in figure 11B to ensure that the vertices stay within a define boundary. The path can also be governed by a rule which applies a curve path (Figure 11C). Another rule can assign the path to be generated dynamically through a stochastic process to provide random perturbations in the movement of the vertex (Figure 11D).
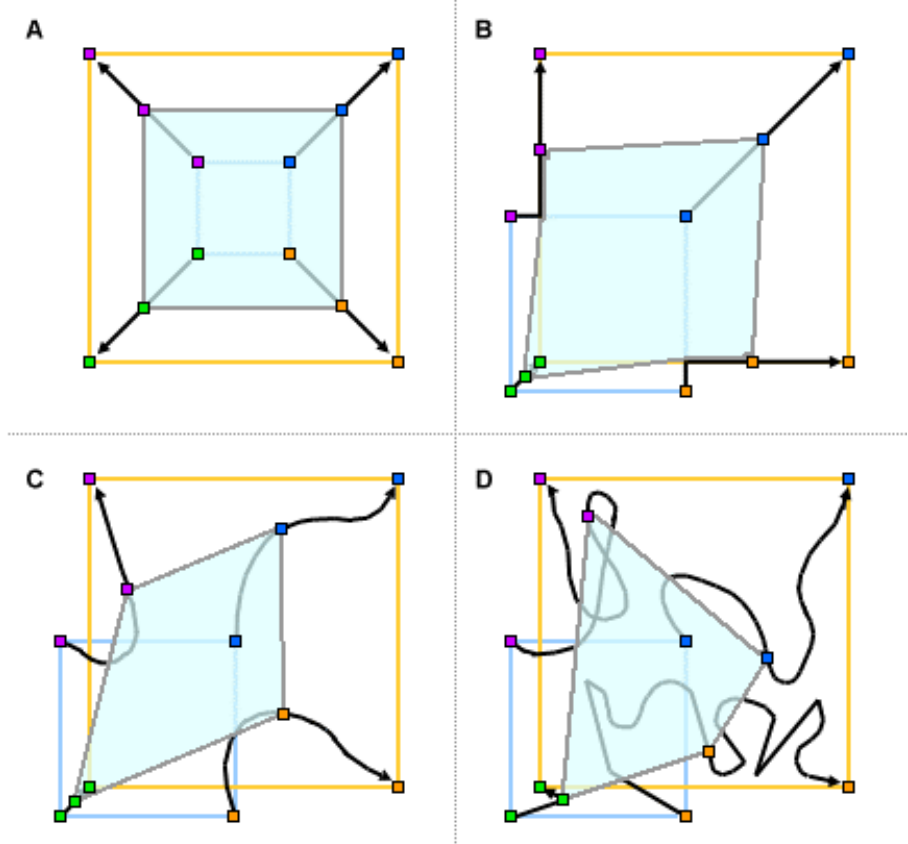

Figure 11 Path variations

\subsection{Trajectory: Rate}

Along with the various paths that a vertex can travel within a bounded area, the rate at which a vertex travels can vary according to another set of parameters. For example, a vertex can travel at a uniform rate, a non-uniform rate or a dynamic rate. A uniform rate of movement means that a vertex moves at a constant rate of speed along a path (Figure 12 Linear). In a non-uniform rate of movement, the vertex moves at a non-constant rate. Exponential progression (Figure 12 Exponential) is another possibility where the vertex initially moves slowly along a path and increases its rate towards the end of its path. An inverse exponential progression would entail a fast initial movement along the path with a slower rate towards the end of the specified time to travel along a path (Figure 12 Inverse Exponential). The rate can be any curve that fits within the time-distance plot. 

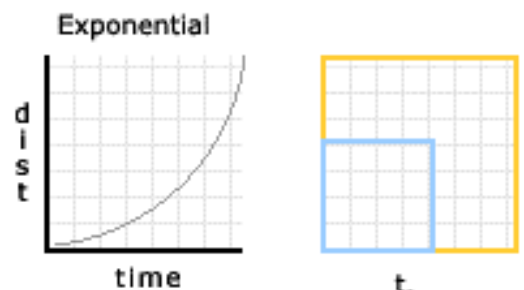

$t_{0}$

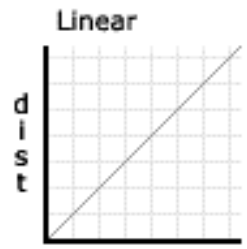

time

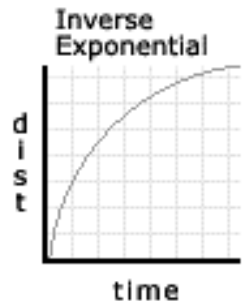

time

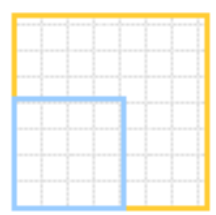

$t_{0}$

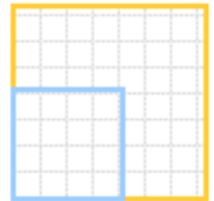

$t_{0}$

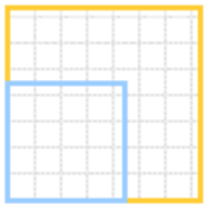

$t_{.33}$

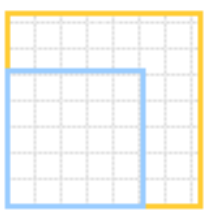

$t_{.33}$

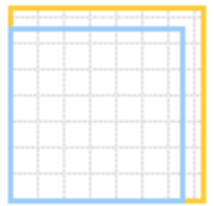

$t_{.33}$

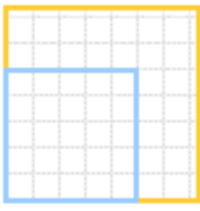

$t_{.66}$

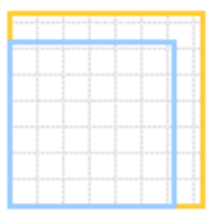

$t_{.66}$

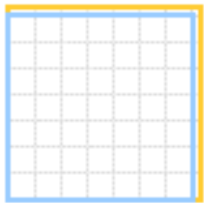

$t_{.66}$

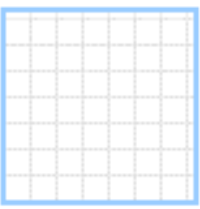

$t_{1}$

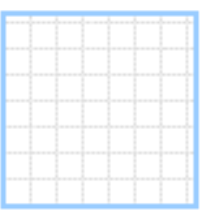

$t_{1}$

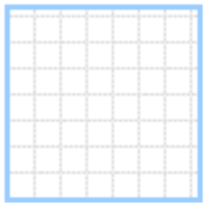

$t_{1}$

Figure 12 Rate variations for transformations along a path or across a space.

Rules governing the tweens can be applied to the entire time-series so that it has one constant rule controlling all the metamorphosis. Another option is to have mixed rules controlling the tweens between each pair of snapshots so that different rules apply to different snapshot pairs within the same time-series. The rules can apply to the correspondence, the path, or the rate along a path for every pair or series. They can also change midstream during a tween to obtain a desired effect using a variety of rule combinations.

\subsection{Spread}

As previously discussed in the background section, Blum's medial axial technique is worth exploring to see if it can provide a better means for representing transformation of geospatial processes. Figure 13 A1 shows the medial axes for the source and target shapes as separate shapes. Below that is figure $13 \mathrm{~B} 1$ displaying a medial axis for a shape representing the combined source and target shapes. The subsequent figures show the initial step (Figure 13 A2 and 13 B2) and the fourth to the last step of how medial axes inform the transformation into the final shape. The benefit of this approach is that the target shape is reflected in the intermediate steps, in other words there is shape persistence between the medial axis and the final target shape. 

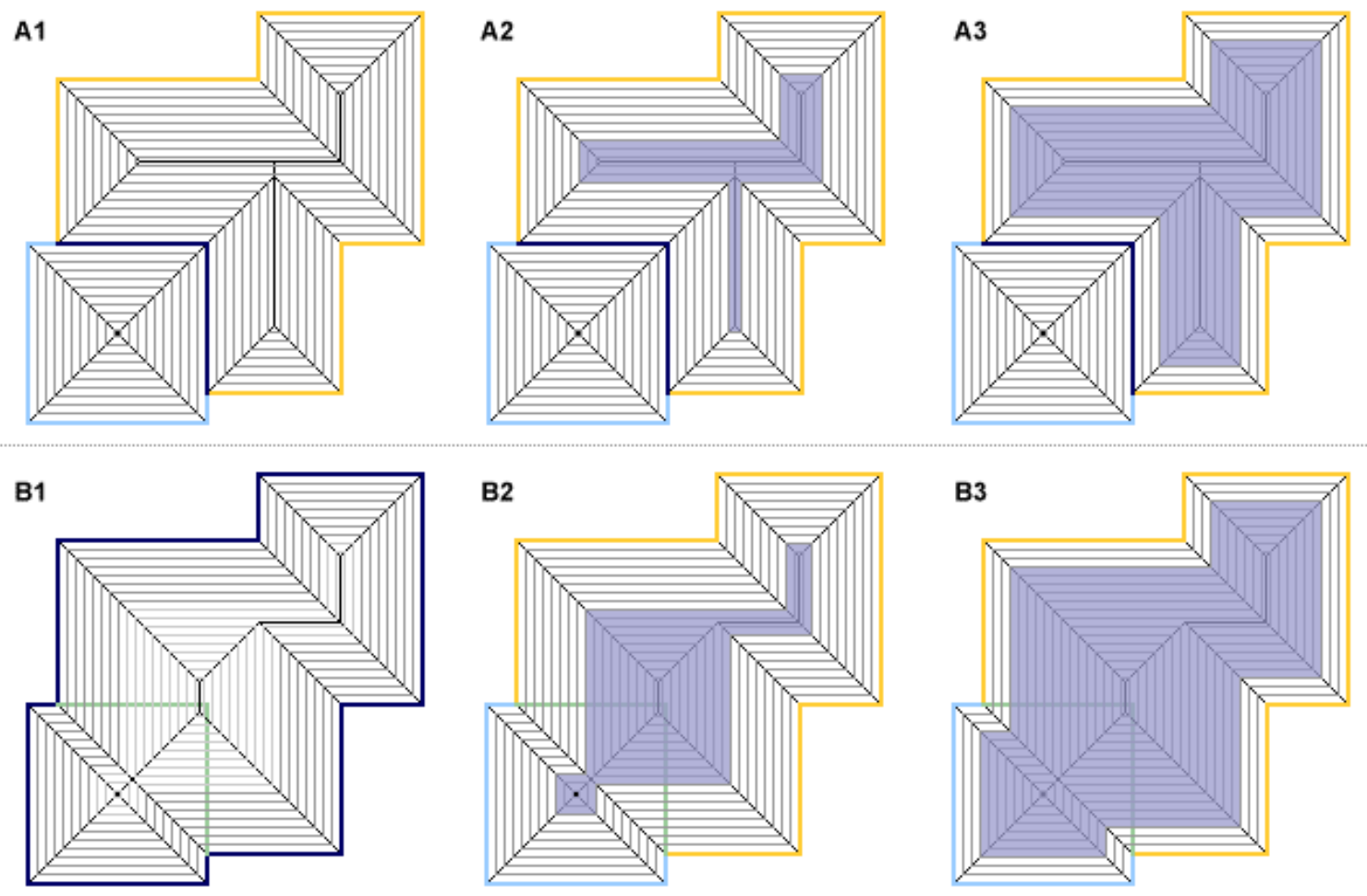

Figure 13 Medial axial of a source and target shape configurations.

In figure 13, row A shows the target shape expanding without any relationship to the source shape. The row consisting of the B figures displays a growth from the medial axis to fill both the source and target shapes. However, this is inconsistent with a tweening process between two shapes because the source shape should diminish whereas the target shape should grow. In addition, the metamorphosis of the source shape to the final shape should initially occur near the shared features. These problems along with another undesired artifact of a medial axis approach to transformations are addressed in the next section.

\section{Results}

Applying the rules and constraints discussed thus far to a geographic process such a wildfire can provide a good comparison between the rules and actual behavior of a geographic process. The different geographic phenomenon may behave (move and transform) in dissimilar manners. For the case of wildfire, the source and target shapes generally have an adjacent with shared feature relationship (Figure 7C), the fire should propagate from a cell to neighboring cell manner. This means that a wildfire will generally spread from the source shape to the target shape starting with the shared feature and morphing into the entire target shape.

The expansion from the medial axis example in figure 13B shows the problem in which the source and target shapes are behaving in the same manner. In actuality, the source shape should diminish over time and the target shape should grow over time. This can be addressed by having the source shape contract one step from the boundary and the target shape expands one step from the medial axes (Figure 14 A1-A3). Still, this approach remains problematic because a wildfire would not instantaneously propagate along the common medial axes of a shape and then expand outwards in the subsequent steps. 

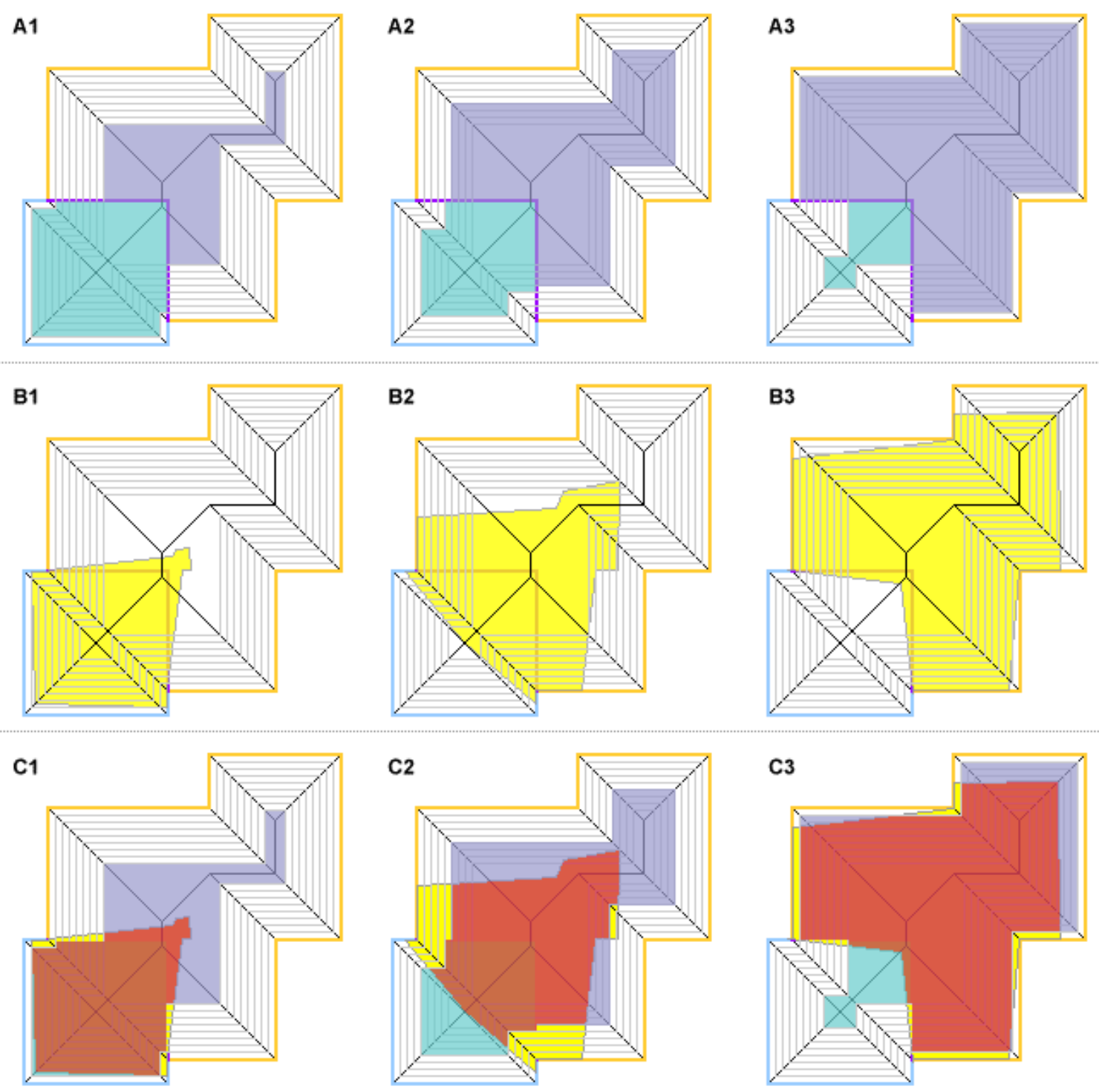

Figure 14 Medial axial, shortest distance and hybrid transformations

One solution is to apply the semi-automatic pseudocode (Figure 14 B1-B3) presented in the methods section and overlay it with the medial axes tweening method in figure 14 A1-A3. This produces an intersecting shape to guide the tweening. An example can be seen in figure $14 \mathrm{C} 1-\mathrm{C} 3$ which depicts a wildfire progression that ensures that the wild fire reaches the entire boundary of the target shape at the same time. This approach is a good compromise between the shortest distance approach and the medial axial approach for better representing a geographic process like wildfire.

\subsection{Grand Prix Fire Case Study}

The Southern California Grand Prix fire of 2003 was used as a case study to apply the constraints presented in the prior section and this was operationalized in Macromedia Flash MX using a manual implementation of the constraints that were previously defined. The fire perimeter data consists of several snapshots and the shape pairs all have shared features in a nested configuration similar to Figure 6B. 
Series A in figure 15 (Figure 15A) shows an ordinary tweening process with inconsistent correspondence of vertices and a lack of adherence to bound ing perimeters resulting in tweened shapes which cross boundaries. Series B (Figure 15B) shows how constraints on shared features reduce artifacts created by shifting vertices, but boundaries are still crossed. The last series (Figure 15C) displays a sequence with constraints on shared features and constraints on boundaries. Compared to the other series, series $\mathrm{C}$ is the most realistic representation of fire progression that follows the known perimeters of the actual fire.

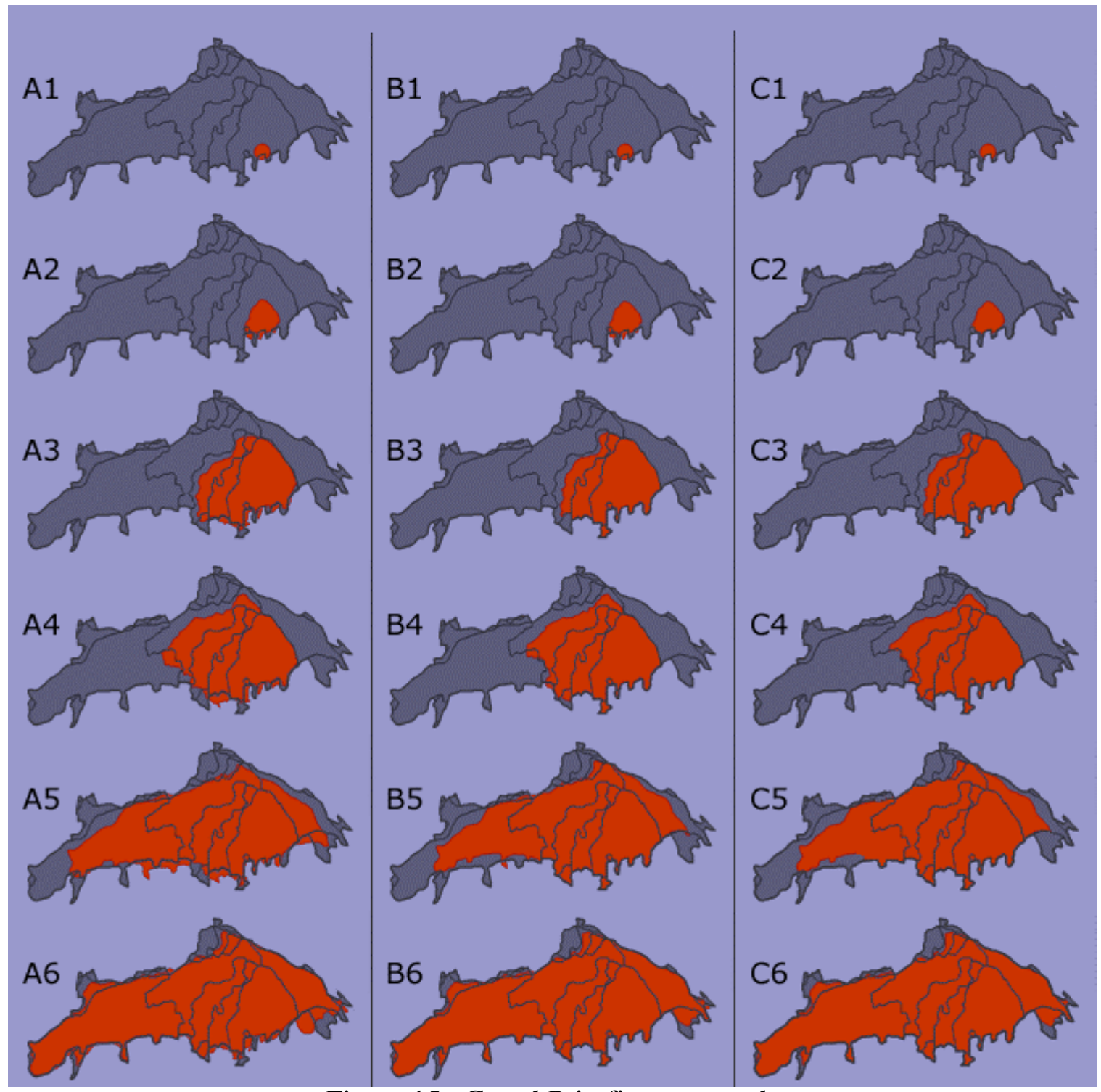

Figure 15. Grand Prix fire case study

The application of basic constraints ensured that the correspondence of points are more realistic in informing the trajectory of vertices. Furthermore, the constraints eliminated the mangling of vertices and prevented tweens from crossing boundaries. The sequence can be performed as an adjacent relationship similar to Figure 6D to provide a more realistic fire progression.

\section{Discussion}


When applying concepts from metamorphosis to geographic dynamics, constraints to inform the tween are often necessary. Unlike the empty space of a computer display, geographic processes have constraints which restrict and guide the movement of entities and events. Identifying different rules and grammars to inform the metamorphosis of snapshot data can assist in the interpolation of in-betweens and improve the visual communication of the underlying phenomenon.

Many varieties of grammars can be formulated to inform behaviors that are specific to a unique phenomenon. The grammars which most realistically represent the change in the context of weather phenomena may differ from those that best represent glacier movement or dynamics. Thus, the important questions to investigate are, "What is the common behavior that these processes share?" and "What fundamental rules will allow users, interested in tweening of snapshot data, to efficiently and effectively produce better tweens?"

The steps involved in producing more realistic tweens are to first identify the shape relationship of the time-series data. Secondly, the correspondence between vertices on the source and target shapes should be determined. Rules pertaining to the trajectory, (path and rate) of travel for the vertices should be applied to obtain a more realistic tween of snapshots.

Procedure for informing the tweening process;

- Shape relationships: 1) Nested without shared feature, 2) nested with shared feature, 3) adjacent with shared feature, 4) overlapping or 5) $n$ to $x * n$.

- Correspondence: 1) manual assignment or 2) semi-automatic process.

- Trajectory-Path: 1) Straight line, 2) Poly line, 3) Curve or 4) Dynamic.

- Trajectory -Rate: 1) Uniform or 2) Non- uniform (Exponential, Inverse exp. or Dynamic).

Identifying the rules to inform the tweening of discrete snapshots can assist in the development of simple procedures. This allows for less labor intensive generation of realistic tweens for an analyst or user.

\section{Conclusion}

The goal of this project was to make the interpolation between two snapshots of discrete entities more effective and efficient. A generalized set of rules was presented to better represent change in a geographical context and possibly improve and inform metamorphosis techniques in other subsequent disciplines. A taxonomy was also described of the different source and target shape relationships followed by an exploration of a semi-automatic correspondence and trajectory rules as well as path and rate variations. A hybrid tweening procedure that relies on a medial axis and semiautomatic transformation approach was presented including the application to a wildfire case study.

Tweening methods that are better at representing underlying geospatial processes can reduce the amount of time and manual labor required to interpolate between two snapshots. A standard set of rules can provide an analyst with tools to rapidly explore tweens that are more appropriate for the data and the phenomenon being visualized. Furthermore, dynamic geospatial processes can be identified and classified along with the appropriate geocomputational algorithms to tween discrete data in an effective and 
efficient manner. Perhaps, research along this path will shed light in to the automatic vertex mapping (correspondence) and trajectory problems, resulting in rules that are specific to each combination of data type and geographic phenomenon that is being represented.

\section{Acknowledgements}

This research was supported by the National Science Foundation under grant \#0416300.

\section{References}

AvRuskin, G. A., Jacquez, G. M., Meliker, J. R., Slotnick, M. J., Kaufmann, A. M., and Nriagu, J. O. (2004). "Visualization and exploratory analysis of epidemiologic data using a novel space time information system." International Journal of Health Geographics, 3(26).

Bertalmio, M., Sapiro, G., and Randall, G. "Morphing active contours: a geometric approach to topology-independent image segmentation and tracking." Proceedings. 1998 International Conference on Image Processing, Chicago, IL, USA, 318-322.

Blum, H. (1967). "A transformation for extracting new descriptors of form." Models for the Perception of Speech and Visual Form, W. Whaten-Dunn, ed., MIT Press, Cambridge, MA, 362-380.

Blum, H., and Nagel, R. (1978). "Form description using weighted symmetric axis features." Pattern Recognition, 10, 167-180.

Bradley, E., and Stuart, J. M. (2001). "Optimization and Human Movement."

Catmull, E. "The problems of computer-assisted animation." Proceedings of the 5th annual conference on Computer graphics and interactive techniques, ACM SIGGRAPH Computer Graphics.

Che, W., Yang, X., and Wang, G. (2004). "Skeleton-driven 2D distance field metamorphosis using intrinsic shape parameters." Graphical Models, 66, 102126.

DeCarlo, D., and Metaxas, D. "Adaptive Shape Evolution Using Blending." Fifth International Conference on Computer Vision, Proceedings ICCV '95, 834-839.

DeCarlo, D., and Metaxas, D. (1998). "Shape Evolution with Structural and Topological Changes using Blending." IEEE Transactions on Pattern Analysis and Machine Intelligence, 20(11), 1186-1205.

Di Fiore, F., Schaeken, P., Elens, K., and Van Reeth, F. "Automatic In-betweening in Computer Assisted Animation by Exploiting 2.5D Modelling Techniques." IEEE $C A$.

Finney, M. A. (1998). "FARSITE: Fire Area Simulator- Model Development and Evaluation." RMRS-RP-4.

Gao, P., and Sederberg, T. W. (1998). "A work minimization approach to image morphing." The Visual Computer, 14(8-9), 390-400.

Glassner, A. (2003). "DMorph." Computer Graphics and Applications, IEEE.

Goodchild, M. F. (1984). "Geocoding and Geosampling." Spatial Statistics and Models, G. L. G. a. C. J. Willmott, ed., Reidel Publishing Company, Dordrecht, Holland, 33-53.

Gotsman, C., and Surazhsky, V. (2001). "Guaranteed intersection-free polygon morphing." Computers \& Graphics, 25, 67-75.

Gueziec, A. (1999). "Locally Toleranced Surface Simplification." IEEE Transactions on Visualization and Computer Graphics, 5(2). 
Johan, H., Koiso, Y., and Nishita, T. "Morphing Using Curves and Shape Interpolation Techniques." Pacific Graphics 2000, Hong Kong, China.

Johnson, I. (1999). "Mapping the fourth dimension: the TimeMap project." 750. 21.

Katz, R. A., and Pizer, S. M. (2003). "Untangling the Blum Medial Axis Transform." International Journal of Computer Vision, 55(2-3), 139-153.

Kerckhove, M. (1999). " A Shape Metric for Blum Ribbons." Journal of Mathematical Imaging and Vision, 11(2), 137-176.

Kim, T. H., Cova, T. J., and Brunelle, A. (submitted 12/2003). "Exploratory map animation for post-event analysis of wildfire protective action recommendations." Natural Hazards Review.

Kirkpatrick, D. G., and Radke, J. D. (1985). "A Framework for Computational Morphology." Computational Geometry, G. T. Tousaint, ed., Elsevier Science Publishers, North Holland, 217-249.

Langran, G. (1993). Time in Geographic Information Systems, Taylor \& Francis, London.

Lee, S., Wolberg, G., and Shin, S. Y. (1998). "Polymorph: morphing among multiple images." Computer Graphics and Applications, IEEE, 18(1), 58 - 71.

Lee, S. Y., Chwa, K. Y., Hahn, J., and Shin, S. Y. (1996). "Image Morphing Using Deformation Techniques."

Lobben, A. (2003). "Classification and Application of Cartographic Animation." The Professional Geographer, 55(3), 318-328.

Martyn, T. "A new approach to morphing 2D affine IFS fractals." Computers \& Graphics, 28(2), 249-272.

Noh, J.-y., and Neumann, U. (1998). "A Survey of Facial Modeling and Animation Techniques." 99-705, USC, Los Angeles.

Pasko, G., Pasko, A., Ikeda, M., and Kunii, T. "Bounded Blending Operations." International Conference on Shape Modeling and Applications 2002 (SMI'02), Banff, Canada.

Pasko, G., Pasko, A., and Kunii, T. (1999). "Space-time blending." 1.

Peuquet, D. J. (2002). Representations of space and time., Guilford, New York.

Reeves, W. T. (1981). "Inbetweening for Computer Animation Utilizing Moving Point Constraints." Computer Graphics and Applications, IEEE, 15(3), 263-269.

Rothermel, R. C. (1972). "A mathematical model for predicting fire spread in wildland fuels."

Sederberg, T. W., Gao, P., Wang, G., and Mu, H. "2D Shape Blending: An Intrinsic Solution to the Vertex Path Problem." Proceedings of Siggraph, Computer Graphics, 15-18.

Shapira, M., and Rappoport, A. (1995). "Shape blending using the star-skeleton representation." Computer Graphics and Applications, IEEE, 15(2), 44-50.

Shih, W. S. V., Lin, W. C., and Chen, C. T. (1998). "Morphologic field morphing: Contour model guided image interpolation." International Journal of Imaging Systems and Technology, 8(5), 480-490.

Stefanidis, A., Eickhorst, K., Agouris, P., and Partsine velos, P. "Modeling and comparing change using spatiotemporal helixes." Proceedings of the 11th ACM international symposium on Advances in geographic information systems, 86-93.

Stiny, G., and Gips, J. (1972). "Shape Grammars and the Generative Specification of Painting and Sculpture." Information Processing 71, 1460-1465.

Surazhsky, T., Surazhsky, V., Barequet, G., and Tal, A. (2001). "Blending polygonal shapes with different topologies." Computers \& Graphics, 25, 29-39.

Thrower, N. (1959). "Animated cartography." The Professional Geographer, 11(6), 9-12. 
Torsello, A., and Hancock, E. R. (2004). "A skeletal measure of 2D shape similarity." Computer Vision and Imape understanding, 95, 1-29.

Treece, G., Prager, R., and Gee, A. (2001). "Volume-based three-dimensional metamorphosis using sphere-guided region correspondence." The Visual Computer, 17(7), 397-414.

Treece, G. M., R.W. Prager, Gee, A. H., and Berman, L. (1999). "Surface interpolation from sparse cross-sections using region correspondence."

Van den Bergh, J., Di Fiore, F., Claes, J., and Van Reeth, F. "Interactively Morphing Irregularly Shaped Images Employing Subdivision Techniques." SIACG 2002.

Winnemöller, H., and Bangay, S. "SUPER-REALISTIC RENDERING USING REALTIME TWEENING." Paper presented at the South African Telecommunication Networks \& Applications Conference, SATNAC, 2001, Wild Coast Sun, South Africa.

Yang, W., Feng, J., Jin, X., Peng, Q., and Forrest, A. R. (2004). "2-D Shape Blending based on Visual Feture Decomposition."

Yuan, M. (1999). "Use of a three-domain representation to enhance GIS support for complex spatiotemporal queries." Transactions in GIS, 3(2), 137-160. 